\title{
Electrode array design determines scalar position, dislocation rate and angle and postoperative speech perception
}

\author{
Manuel Christoph Ketterer ${ }^{1}$ (1) $\cdot$ Antje Aschendorff ${ }^{1} \cdot$ Susan Arndt $^{1} \cdot$ Rainer Beck $^{1}$
}

Received: 29 June 2021 / Accepted: 26 October 2021 / Published online: 15 November 2021

(c) The Author(s) 2021

\begin{abstract}
Purpose The aim of this study is to examine the scalar dislocation rate in straight and perimodiolar electrode arrays in relation to cochlear morphology. Furthermore, we aim to analyze the specific dislocation point of electrode arrays depending on their design and shape and to correlate these results to postoperative speech perception.

Methods We conducted a comparative analysis of patients (ears: $n=495$ ) implanted between 2013 and 2018 with inserted perimodiolar or straight electrode arrays from Cochlear ${ }^{\mathrm{TM}}$ or MED-EL. CBCT (cone beam computed tomography) was used to determine electrode array position (scalar insertion, intra-cochlear dislocation, point of dislocation and angular insertion depth). Furthermore, cochlear morphology was measured. The postoperative speech discrimination was compared regarding electrode array dislocation, primary scalar insertion and angular insertion depth.

Results The electrode array with the highest rate of primary SV insertions was the CA; the electrode array with the highest rate of dislocations out of ST was the Flex ${ }^{\text {Soft }}$. We did not find significantly higher dislocation rates in cochleostomy-inserted arrays. The angle of dislocation was electrode array design-specific. A multivariate nonparametric analysis revealed that the dislocation of the electrode array has no significant influence on postoperative speech perception. Nevertheless, increasing angular insertion depth significantly reduced postoperative speech perception for monosyllables.

Conclusion This study demonstrates the significant influence of electrode array design on scalar location, dislocation and the angle of dislocation itself. Straight and perimodiolar electrode arrays differ from each other regarding both the rate and place of dislocation. Insertion via cochleostomy does not lead to increased dislocation rates in any of the included electrode arrays. Furthermore, speech perception is significantly negatively influenced by angular insertion depth.
\end{abstract}

Keywords Cochlear morphology $\cdot$ Electrode array design $\cdot$ Scalar position $\cdot$ Coverage $\cdot$ Speech perception

\section{Abbreviations}

ST Insertion in scala tympani

SV Insertion in scala vestibuli

TD Tympani dislocation $=$ dislocation out of ST

VD Vestibuli dislocation $=$ dislocation out of SV

CBCT Cone beam computed tomography

HRCT High-resolution computed tomography

CI Cochlear implant/cochlear implantation

CA Cochlear ${ }^{\mathrm{TM}}$ Contour Advance ${ }^{\circledR}$ electrode array

SMA Cochlear ${ }^{\mathrm{TM}}$ slim modiolar ${ }^{\circledR}$ electrode array

SSA Cochlear ${ }^{\mathrm{TM}}$ slim straight ${ }^{\circledR}$ electrode array

Manuel Christoph Ketterer

manuel.christoph.ketterer@uniklinik-freiburg.de

1 Department of Otorhinolaryngology-Head and Neck Surgery, Faculty of Medicine, Medical Center-University of Freiburg, University of Freiburg, Killianstrasse 5, 79106 Freiburg, Germany

$$
\begin{array}{ll}
\text { Flex }^{24} & \text { MED-EL Flex } \\
\text { Flex }^{28} & \text { electrode array } \\
\text { Flex }^{\text {Soft }} & \text { MED-EL Flex } \\
\text { MEL } & \text { electrode array } \\
\text { RW } & \text { Round electrode array } \\
\text { ERW } & \text { Extended round window } \\
\text { CS } & \text { Cochleostomy }
\end{array}
$$

\section{Introduction}

Cochlear implant (CI) surgery focusses more and more on the impact of cochlear morphology and consequently on intra-cochlear electrode array position and postoperative speech perception. Previous studies described different ways of estimating cochlear morphology preoperatively. Escudé et al. [1] established distance $\mathrm{A}$ as the distance from the round window $(=\mathrm{RW})$ to the lateral wall and a perpendicular distance B, both intersecting the modiolus. Ketterer et al. [2] 
described a third measure, the cochlear height and the fact that the electrode array was more likely to dislocate within a cochlea of smaller height and smaller diameter. Aschendorff et al. [3] first examined scalar position via rotational tomography for patients inserted with a Contour $(n=21)$ versus a Contour Advance $(n=22)(=\mathrm{CA})$ electrode array and described significantly higher speech discrimination results for scala tympani (ST) compared to scala vestibuli (SV) insertion. In a linear regression analysis for 14 of the 15 patients described by Skinner et al. [4], Finley et al. [5] calculated that scalar position, age at implantation and total number of electrode contacts within the SV accounted for $83 \%$ of the variance in monosyllabic word scores. Holden et al. [6] described that the position of electrode arrays closer to the modiolus was positively correlated with the outcome. As a result, scalar position detection of the electrode array via CBCT (cone beam computed tomography) or HRCT (high resolution computed tomography) should be a consideration in postoperative quality control to provide important feedback to the surgeon [3]. The goal of this study is to examine the scalar dislocation rate in both straight and perimodiolar electrode arrays. To the best of our knowledge, this is the first large cohort study analyzing the specific position of dislocation of electrode arrays depending on their design and shape. Furthermore, we aimed to evaluate the impact of scalar dislocation, electrode array design and angular insertion depth on postoperative speech perception.

\section{Methods}

\section{Study and subject}

We performed a retrospective analysis of adult patients implanted between 2013 and 2018. HRCT and magnetic resonance imaging had been conducted preoperatively and patients with cochlear anomalies and signs of sclerosis or obliteration were excluded from this study. We only included patients inserted with a Cochlear ${ }^{\mathrm{TM}}$ Contour Advance $^{\circledR}$ (CI24RECA, CI412/512/612) (=CA), Cochlear ${ }^{\mathrm{TM}}$ slim straight ${ }^{\circledR}(422 / 522 / 622)\left(=\right.$ SSA) or Cochlear ${ }^{\mathrm{TM}}$ slim modiolar $^{\circledR}$ (532/632) electrode array (= SMA) (Cochlear Limited, NSW, Sydney, Australia) and MED-EL Flex ${ }^{24}$, MED-EL Flex ${ }^{28}$ and MED-EL Flex ${ }^{\text {Soft }}$ (MED-EL, Innsbruck, Austria). Electrode arrays were inserted via cochleostomy $(=\mathrm{CS})$, round window $(=\mathrm{RW})$ and extended round window (=ERW) insertion. The patient chose the manufacturer following individual consulting. If the patient chose MED EL and showed residual hearing, the FLEX ${ }^{24}$ was used in most cases. In patients implanted with a device from Cochlear ${ }^{\mathrm{TM}}$, the SSA or the SMA was used in patients with residual hearing, otherwise the CA was also used quite often due to the later availability of the SSA and the SMA.

\section{Radiological evaluation}

Postoperative imaging was performed using a DynaCTequipped Axium Artis dTA angiography unit (Siemens Co., Erlangen, Germany) with a digital flat-panel detector [3, 7]. Two experienced head and neck surgeons and two head and neck radiologists independently analyzed the scans regarding scalar electrode position (ST versus SV insertion, intracochlear dislocation, angular insertion depth) and cochlear size (diameters in length and width) and used Impax 6 from Agfa Healthcare for reconstruction. The scans were not evaluated by the surgeons who, performed the CI surgery but by independent and experienced head and neck surgeons to reduce bias. All included electrode arrays were fully inserted. Cochlear size was evaluated in distance A from the round window to the lateral wall through the modiolus and perpendicular distance $\mathrm{B}[1,2,8]$. The angular insertion depth was evaluated between the vectors of distance A and the distance through the bloom artefact of the apical electrode and the modiolus as described before [2, 8, 9]. Dislocation analysis and analysis of scalar position were performed on three-dimensionally reconstructed cross-sectional images as previously described $[2,8]$, i.e. the 3D-reconstruction could be rotated and browsed in whichever direction the specialists needed to come to their respective conclusion. Every image with discrepancy was reviewed and discussed interdisciplinary until a final agreement and measurement was achieved.

We compared preoperative HRCT scans to postoperative CBCT scans to examine the hypothesis that straight electrode arrays could lead to a mismatch of cochlear morphology measurements due to their more lateral electrode artifacts.

\section{Audiological evaluation}

Open set speech perception is evaluated regularly in a soundproof chamber in a standard clinical setting using the Freiburg numbers and the Freiburg monosyllables test both with presentation at a volume of $65 \mathrm{~dB}$ SPL in quiet. Speech discrimination is scored as percentage correct. The audiologists conducting speech perception were blinded and did not know scalar position or dislocation analysis.

\section{Statistics}

Statistical analysis was performed using Gnu R statistical computation and graphics system (GNU R, Version 3.6.2, Core Team, Vienna, Austria, http://www.R-project.org), extended with the packages NLME (Linear and Nonlinear Mixed Effects Models, Version 3.1, Pinheiro et al., https:// CRAN.R-project.org/package $=$ nlme $)$ and ggplot $2($ Version 
3.3.1, Hadley Wickham, https://ggplot2.tidyverse.org). Where applicable, ANOVA and Tukey's Honest were used. Nonlinear mixed effect models were applied for the analysis of speech discrimination and compared directly by ANOVA and AIC. For array comparisons, the residuals were analyzed using pairwise Mann-Whitney $U$ tests with adjustment by Holm. Results were calculated descriptively and are shown in the text and in tables as mean, standard deviation, maximum and minimum. The level of significance was set at $5.0 \%$.

\section{Ethics committee}

This retrospective study took place in the Department of Otorhinolaryngology, Head and Neck Surgery at the Implant Center of the University Hospital Freiburg. The study was approved by the hospital's Ethics Committee according to the Declaration of Helsinki (Washington, 2002) (Number of Ethics Committee approval: 406/19) and registered in

Table 1 Synopsis of study group (in total: $n=495$ )

\begin{tabular}{|c|c|}
\hline Manufacturer $(n)$ & $\begin{array}{l}\text { Cochlear }{ }^{\mathrm{TM}}: 327 \\
\text { MED-EL: } 168\end{array}$ \\
\hline Electrode array $(n)$ & $\begin{array}{l}\text { Contour Advance }\left(\text { Cochlear }^{\mathrm{TM}}\right) \\
\text { (=CA): } 143 \\
\left.\text { CI 422/522/622 (Cochlear }{ }^{\mathrm{TM}}\right) \\
\text { (=SSA): } 162 \\
\left.\text { CI 532/632 (Cochlear }{ }^{\mathrm{TM}}\right) \\
\quad \text { (=SMA): } 22 \\
\text { Flex }^{24} \text { (MED-EL): } 129 \\
\text { Flex }^{28} \text { (MED-EL): } 24 \\
\text { Flex }^{\text {Soft }} \text { (MED-EL): } 15\end{array}$ \\
\hline Side $(n)$ & $\begin{array}{l}\text { Left: } 259 \\
\text { Right: } 236\end{array}$ \\
\hline Age & 52.7 years $(\min 18.0 ; \max 86.2)$ \\
\hline
\end{tabular}

the German Clinical Trials Register (http://www.drks.de/ DRKS00019807).

\section{Results}

\section{Study, subject and cochlear morphology}

We included 495 ears implanted between 2013 and 2018. We included 40 bilaterally implanted and 415 unilaterally implanted patients. 259 left and 236 right cochleae; 327 ears, implanted with a device from Cochlear ${ }^{\mathrm{TM}}$ and 168 ears implanted with a device from MED-EL were included. The mean age was 52.7 years and the most-often inserted electrode array was the SSA with $32.7 \%$ (see Table 1: distribution of analyzed electrode arrays). The measurements of the diameters of the cochlear basal turn confirm previous studies [1, 2], calculating mean distance A with $9.92 \mathrm{~mm}$ and distance B with $6.74 \mathrm{~mm}$ (see Table 2). Regarding the electrode array portfolio of Cochlear ${ }^{\mathrm{TM}}$, the cochlear basal turn size (product of distance A and B) shows significant impact on the surgeon's electrode array choice (see Fig. 1). Differences between the two manufacturers regarding the cochlear basal turn product were not analyzed due to patient's preoperative choice of the manufacturer. The use of the CA is significantly more frequent in cochleae with a smaller cochlear basal turn product of distance $\mathrm{A}$ and $\mathrm{B}$ compared to the SMA and the straight electrode (SSA) array of Cochlear ${ }^{\mathrm{TM}}$ (CA versus SSA: $p<0.0001$; SMA versus SSA: $p=0.0025$ ). Nevertheless, we did not find significance for the cochlear basal turn product between the different electrode arrays of MED-EL.

We excluded the hypothesis that the SSA could lead to larger cochlear measurements due to the lateral electrode array artifacts. Therefore, we compared preoperative HRCT
Table 2 Cochlear measurements (distance $\mathrm{A}$ and $\mathrm{B}$ ), insertion angle, scalar position in total ( $\mathrm{SD}=$ standard deviation $)$ and distribution of the insertion technique

\begin{tabular}{|c|c|c|c|c|}
\hline & Mean & SD & Minimum & Maximum \\
\hline Distance A (mm) & 9.92 & 0.86 & 7.4 & 12.2 \\
\hline Distance B (mm) & 6.74 & 0.49 & 5.4 & 8.1 \\
\hline Insertion angle $\left(^{\circ}\right)$ & 418.8 & 103.7 & 199 & 794 \\
\hline Scalar position in total $(n)$ & $\begin{array}{l}\text { ST: } 434(87.7 \%) \\
\text { TD: } 32(6.5 \%) \\
\text { SV: } 25(5 \%) \\
\text { VD: } 4(0.8 \%)\end{array}$ & & & \\
\hline \multirow{7}{*}{$\begin{array}{l}\text { Insertion technique in total }(n) \\
\text { and percentage }(\%)\end{array}$} & Electrode array & $\mathrm{CS}$ & RW & ERW \\
\hline & $\mathrm{CA}\left(\right.$ Cochlear $\left.^{\mathrm{TM}}\right)$ & $140 / 98 \%$ & $3 / 2 \%$ & I \\
\hline & SSA $\left(\right.$ Cochlear $\left.{ }^{\mathrm{TM}}\right)$ & $47 / 29 \%$ & $110 / 68 \%$ & $5 / 3 \%$ \\
\hline & SMA (Cochlear $\left.{ }^{\mathrm{TM}}\right)$ & $12 / 54.5 \%$ & $10 / 45.5 \%$ & l \\
\hline & Flex $^{24}$ (MED-EL) & $21 / 16.3 \%$ & $102 / 79.2 \%$ & $6 / 4.2 \%$ \\
\hline & Flex $^{28}$ (MED-EL) & $10 / 41.6 \%$ & $13 / 54.2 \%$ & $1 / 4.2 \%$ \\
\hline & Flex $^{\text {Soft }}$ (MED-EL) & $12 / 80 \%$ & $3 / 20 \%$ & 1 \\
\hline
\end{tabular}

$C S$ cochleostomy, $R W$ round window, $E R W$ extended round window 
Fig. 1 Left: Regarding electrode arrays from Cochlear ${ }^{\mathrm{TM}}$ $(\mathrm{CA}=$ Contour Advance; $\mathrm{SMA}=$ CI 532/632 $=$ slim modiolar array; $\mathrm{SSA}=\mathrm{CI}$ 422/522/622= slim straight array) the use of the CA is significantly more frequent in cochleae with a smaller size of the cochlear basal turn (product of distance A and B). Right: We could not find significance for the cochlear basal turn product between the different electrode arrays of MED-EL

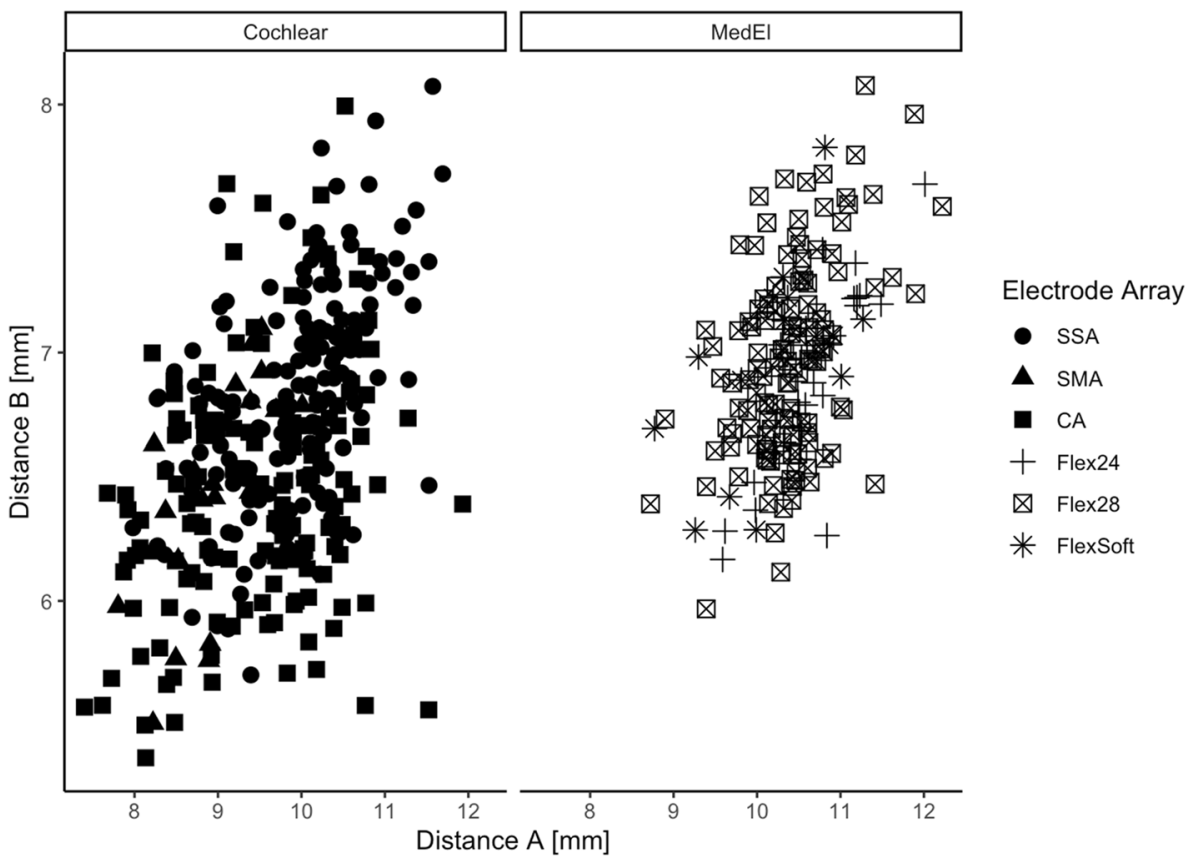

scans to postoperative CBCT scans. We evaluated both scans blinded and independently and did not find different measurements for either distance A or B.

Furthermore, we compared cochlear size to dislocation behavior and could not detect significant differences for cochlear distance A or B compared dislocated or non-dislocated electrode arrays.

\section{Angular insertion depth and dislocation manner}

Figure 2 shows the mean angular insertion depth for each included electrode array. Regarding the included Cochlear ${ }^{\mathrm{TM}}$ electrode arrays, we measured a significantly higher angular insertion depth for the SSA compared to the CA $(p=0.0004)$. The angular insertion depth was comparable between the CA and the SMA ( $p=0.15)$ or between the SSA and the SMA ( $p=0.9996)$. Regarding the electrode arrays from MED-EL, the Flex ${ }^{24}$ showed significantly lower angular insertion depth compared to the longer electrode arrays Flex $^{28}$ and Flex ${ }^{\text {Soft }}(p<0.00001)$ as expected. All included electrode arrays from Cochlear ${ }^{\mathrm{TM}}$ showed significantly shorter angular insertion depth than the electrode arrays from MED-EL ( $p$ in all comparisons <0.0065) (see Fig. 2).

In regard to insertion types, the electrode arrays behaved distinctively in this cohort: CA exhibited the highest rate of SV insertions [SV: 16.1\%; TD (dislocation out of ST): $15.4 \%]$; the electrode array with the highest rate of ST dislocations was the Flex ${ }^{\text {Soft }}$ (SV 6.7\%; TD 20.0\%). The SMA showed no dislocations; the SSA only one dislocation out of ST and $3 \mathrm{SV}$ insertions via CS (SV 1.9\%; TD 0.6\%) (see Fig. 3). An SV insertion was defined as a direct insertion into SV. SV insertions mostly occurred in CS approaches and most frequently for the CA electrode array (see Fig. 3). As SV insertions mostly depend on the position of the CS, they are only partially influenced by array design.

Table 2 and Fig. 3 show that electrode arrays included in this study were inserted via CS and RW and in some cases via ERW. Comparing angular insertion depth in all approaches (ANOVA Tukey post-hoc), we could not find any significant impact.

Measuring the specific position of dislocation for each electrode array, we found that the position of dislocation depends on the electrode array itself (see Fig. 4). We measured a significant lower point of dislocation for the CA compared to both the Flex ${ }^{28}(p<0.00001)$ and the Flex ${ }^{\text {Soft }}$ $(p<0.00001)$. The point of dislocation is electrode-design specific. Perimodiolar electrode arrays dislocate between 160 and $180^{\circ}\left(\mathrm{CA}:\right.$ mean $\left.\pm \mathrm{SD}: 170 \pm 25^{\circ}\right)$, whereas straight electrode arrays dislocate between $280^{\circ}$ and $330^{\circ}\left(\right.$ Flex $^{28}$ : mean \pm SD: $284 \pm 87^{\circ}$; Flex $^{\text {Soft. }}$ mean \pm SD: $\left.330 \pm 36^{\circ}\right)$.

\section{Impact on speech perception}

Data could not be acquired for 11 ears for the evaluation of speech discrimination, therefore 484 ears were included. The Freiburg Number discrimination test showed a considerable ceiling effect over all measurements in all patients (1st quartile $80 \%$, mean $82.25 \%$, 3rd quartile $100 \%$ ) and was therefore discarded in regard to further analysis. The Freiburg Monosyllable test showed a more homogeneous distribution (1st quartile $20 \%$, mean $43.9 \%$, 3rd quartile $70 \%$ ). After constructing an asymptotic growth model for every individual ear, the 


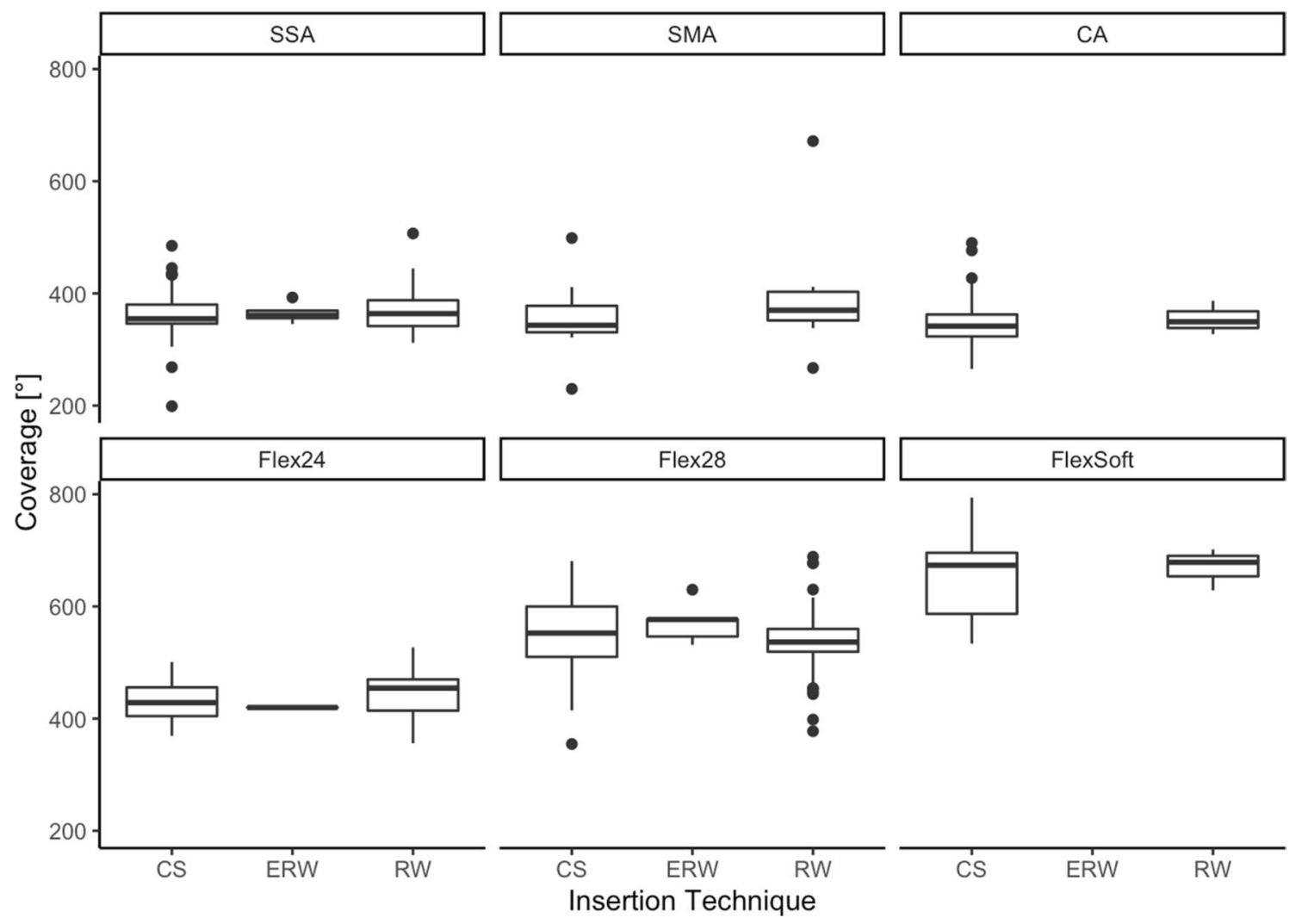

Fig. 2 Angular insertion depth depends on electrode array design and correlates to electrode array length. Insertion technique has no significant influence on electrode array-specific angular insertion depth $(\mathrm{CS}=$ cochleostomy; $\mathrm{RW}=$ round window; ERW = extended round window)

fitness of different models could be compared using ANOVA. Including angular insertion depth could improve the fitness of the model significantly when including all electrode arrays $(p<0.0001)$, but not when comparing electrode arrays from Cochlear ${ }^{\mathrm{TM}}$ and MED-EL separately. Pooling all electrode arrays, there was a significant $(p<0.0001)$ but very small effect favoring shorter electrode arrays (see Fig. 5). In regard to primary insertion into SV or ST and in regard to dislocated or non-dislocated electrode arrays, the models showed no higher or lower speech perception results (pooled and manufacturers separately).

Comparing the residual hearing of the different electrode arrays, expressed as PTA $2(250$ and $500 \mathrm{~Hz})$, the tendency towards shorter electrode arrays in patients with better residual hearing can be seen (see Fig. 6). How the surgeon chooses the respective length of the electrode array should be examined further.

\section{Discussion}

\section{Study, subject and cochlear morphology}

This is the largest study ( $n=495$ ears) so far evaluating the influence of cochlear morphology and electrode array design on electrode array position. We measured the cochlear basal turn with a mean distance A of $9.92 \mathrm{~mm}$ and distance B of $6.74 \mathrm{~mm}$ (see Table 2). Once more, we can now confirm the data published so far $[1,2,10]$. Furthermore, this is the first study describing that the CA was more frequently implanted in smaller cochlea with less basal turn size (product of distance A and B) (see Fig. 1). The surgeon usually chooses the CA electrode array in patients without residual hearing to be as close to the modiolus as possible. Furthermore, the CA is usually not the first 

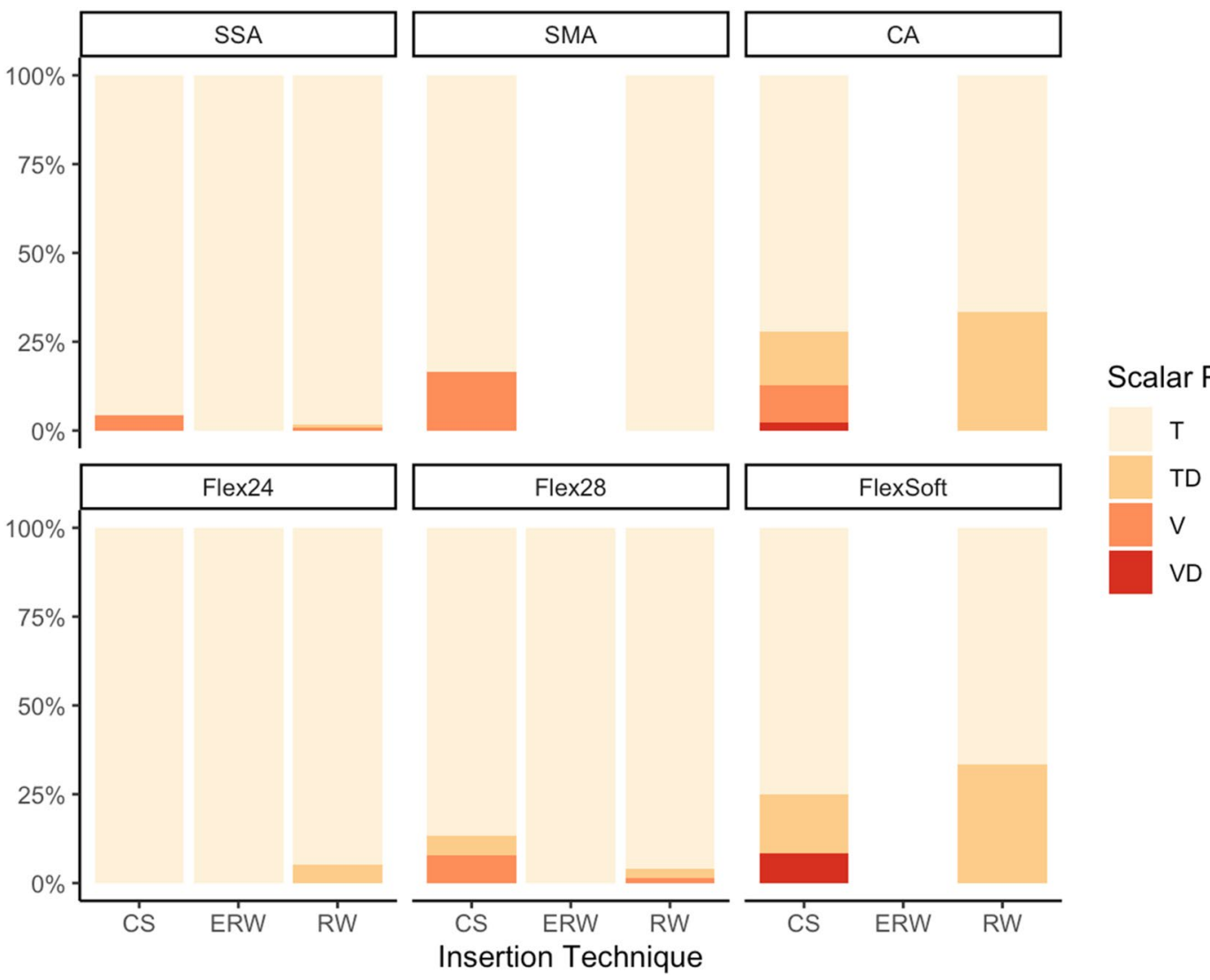

Fig. 3 Count of primary scala tympani insertions (T), dislocations out of ST (TD), scala vestibuli insertions (V) and dislocations out of scala vestibuli (VD) for each examined electrode array

electrode array choice to preserve residual hearing due to its rigidity and larger diameter.

\section{Angular insertion depth and dislocation manner}

We could measure specific angular insertion depth for each included electrode array (see Fig. 2). Previous studies [1, 2] showed that the angular insertion depth is dependent on the cochlear size. This work extends the earlier studies by defining electrode array-specific angular insertion depth, depending on both electrode array design and cochlear morphology.

This study demonstrates specific dislocation behavior of each examined electrode array (see Fig. 3). The electrode array with the highest rate of ST dislocations was the Flex ${ }^{\text {Soft }}$ array. The electrode array with the highest rate of SV insertions was the CA due to insertion via CS. Ketterer et al. [2] showed that dislocation depends on cochlear morphology and that a smaller cochlear height is a risk factor for SV insertion and dislocation for CS-inserted CA electrode arrays. Nevertheless, Aschendorff et al. [11] described individual learning curves of the surgeon and dislocation rates also depend on the surgeon's experience not only on electrode array design and cochlear morphology.
The SMA did not dislocate in any of the included patients in this study. Aschendorff et al. [12] described in a multi-center study that all patients $(n=44)$ implanted with the SMA from Cochlear ${ }^{\mathrm{TM}}$ exhibited a complete ST insertion without dislocation in round window and cochleostomy approaches. We can now confirm that the SMA is the electrode array without any dislocation and seems to be very well designed for staying within the initial inserted cochlear scala.

We could show that the position of dislocation is electrode-design specific (see Fig. 4) and depends on electrode array design itself. The SSA has a stiff internal stylet and is a lateral wall array. Therefore, the dislocation point is more apical than the dislocation point of the perimodiolar CA. The CA is inserted via an Advance Off-Stylet ${ }^{\mathrm{TM}}$ insertion technique and due to its preformed perimodiolar design the point when dislocation might happen is earlier and at approximately $180^{\circ}$. Further studies described the ascending cochlear basal turn at around $180^{\circ}$ as sensitive for scalar dislocation [13-15]. Aschendorff et al. [7] speculated that perimodiolar electrode arrays may touch the outer cochlear wall at $180^{\circ}$ while rotating with an upward 


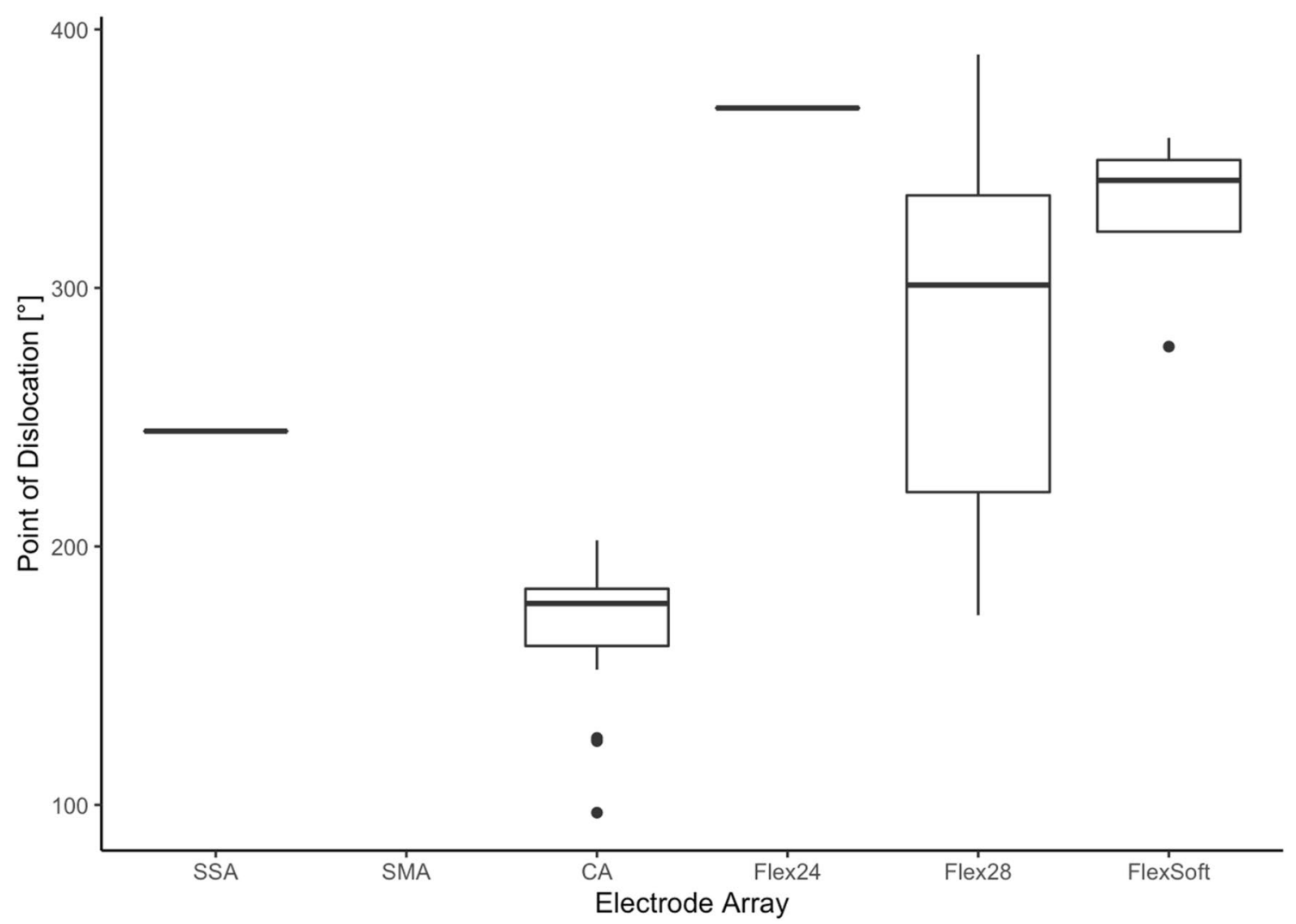

Fig. 4 The angle of dislocation is electrode-array-specific

direction and being pushed forward, resulting in perforation of the basilar membrane.

With a maximal active length of $25 \mathrm{~mm}$ (source: Cochlear $\left.{ }^{\mathrm{TM}}\right)$, the SSA is shorter than the straight Flex ${ }^{28}$ and Flex $^{\text {Soft }}$ and shows less insertion depth. MED EL electrode arrays do not have such a rigid internal stylet as the SSA. Therefore, the point of dislocation is higher in longer and more flexible MED EL Flex ${ }^{28}$ and Flex ${ }^{\text {Soft }}$ due to their flexibility and trajectory. Furthermore, the height of the ST decreases within the ascending part of the basal turn towards the apical cochlear part [16]. Therefore, long electrode arrays like the Flex ${ }^{28}$ and Flex ${ }^{\text {Soft }}$ showed increasing risk of dislocation in the apical cochlear part. The fact that the Flex ${ }^{24}$ showed the highest dislocation point is interesting but since there was only one dislocated Flex ${ }^{24}$ array further studies are required.

Boyer et al. [13] analyzed 61 CBCT scans of 54 patients. Eight perimodiolar electrode arrays and one straight electrode array were described as dislocated. The authors speculated that straight electrode arrays dislocate at approximately $370^{\circ}$, whereas perimodiolar electrode arrays dislocate at around $170^{\circ}-190^{\circ}$. We can now confirm that the CA dislocates at approximately $170^{\circ}$, corresponding to the ascending part of the cochlear basal turn. Boyer et al. [13] compared only two groups: perimodiolar (CI 512 and CI24RECA) versus straight $\left(\right.$ Flex $^{\text {Soft }}$, Flex $^{24}$, Flex $^{28}$ and Flex ${ }^{\text {Standard }}$ ) electrode arrays. Whereas their defined perimodiolar group seems to be a homogeneous electrode array cohort $(n=31)$, the straight electrode array group $(n=30)$ is not only too small to define angular insertion depth and manner of dislocation but also inhomogeneous, comparing electrode arrays from MED-EL of different lengths and diameters. Furthermore, they neither excluded the CS-inserted electrode arrays nor calculated if there is a statistically relevant effect of RW versus CS or not. We examined the influence of the insertion location and can show that CS does not lead to higher dislocation rates or SV insertions in any of the included arrays. Boyer et al. and Wanna et al. [13, 17] described that straight electrode arrays are more often completely inserted within the ST and hypothesized that straight electrode arrays are more flexible due to the silicon density of the electrode array. Nevertheless, they did not exclude CS-inserted electrode arrays. Additionally, the CA electrode array, which was originally designed for CS approach, was inserted via RW. Rebscher et al. and Souter et al. [18, 19] described that the $\mathrm{CA}$ is only designed for CS due to the higher incidence of cochlear trauma in RW approach. They argued that the electrode array may be too close to the lateral wall, which might result in traumatic deflection. Therefore, the insertion of the $\mathrm{CA}$ via $\mathrm{CS}$ is recommended, even though $\mathrm{RW}$ insertions are 


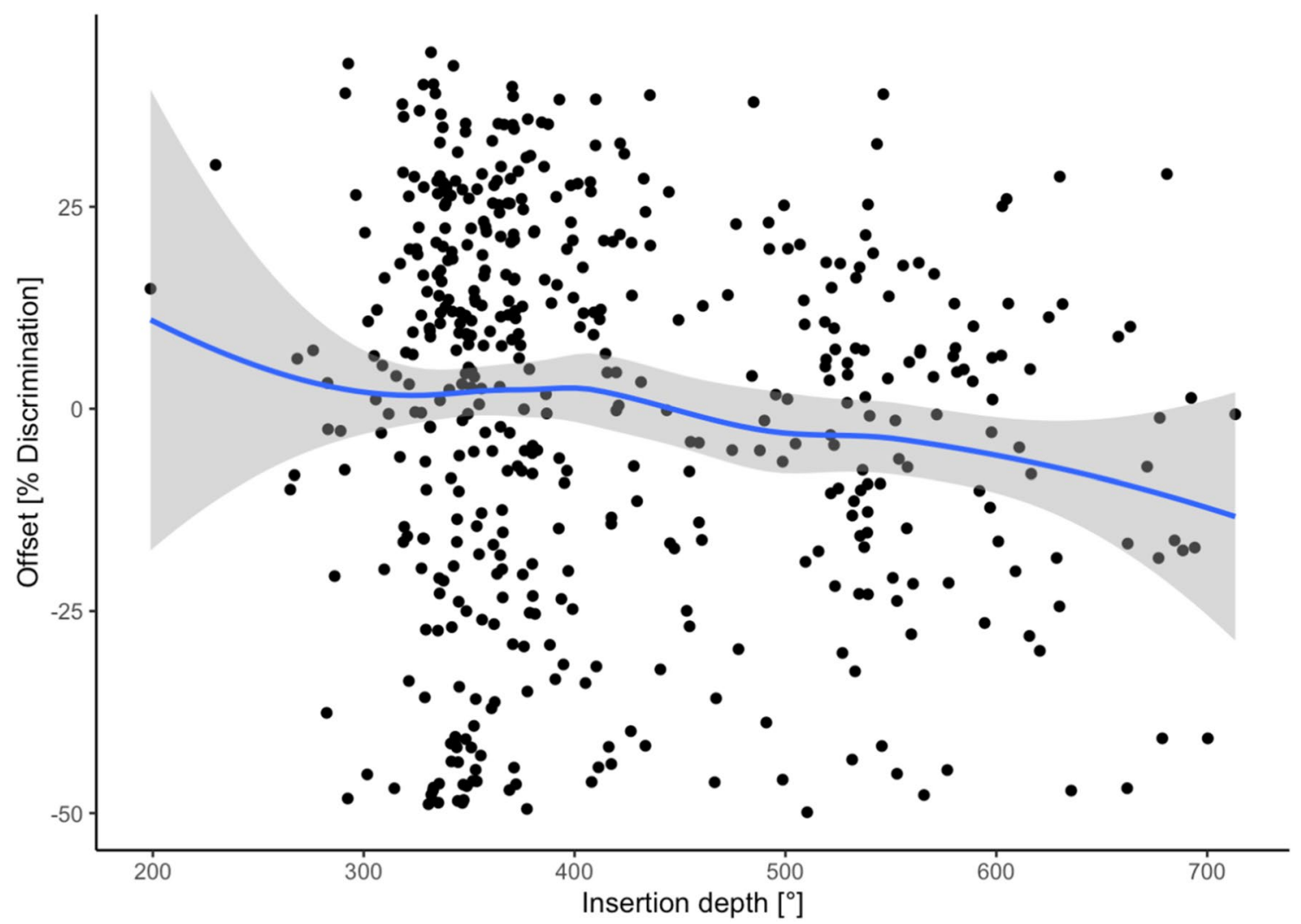

Fig. 5 Plotting individual speech discrimination versus the insertion depth shows a significant negative effect of deeper angular insertion with regard to speech discrimination. The $y$ axis depicts the influence

also possible in exceptions. Table 2 shows that with only a few exceptions, the included CA arrays of our study were not inserted via RW, but in $98 \%$ via CS. In conclusion, this study extends the previous knowledge of angular insertion depth, dislocation behavior and the influence of cochlear morphology. Furthermore, we could measure defined angular insertion depth and dislocation data of each included electrode array and could show that each electrode array has a specific position of dislocation.

\section{Impact on speech perception}

Electrode array design and its influence on speech perception is still a disputed topic. This study demonstrates that speech perception may be negatively influenced by the electrode array's angular insertion depth. Scalar dislocation has no significant impact on postoperative speech perception. Perimodiolar electrode arrays have been described as closer to the spiral ganglion cells with reduced spread of excitation $[12,20]$ and situated closer to the target spiral ganglion cells, therefore requiring lower stimulation levels than the lateral wall straight electrode arrays. That might provide better speech perception results [12]. Holden et al. of angular insertion depth as offset in speech discrimination compared to the model that does not comprise angular insertion depth as a factor

[6] reported that the position of electrode arrays closer to the modiolus was positively correlated with the outcome. Nevertheless, other studies reported lower speech discrimination levels for perimodiolar electrode arrays compared to straight electrode arrays [21, 22]. We did not observe different speech discrimination between the groups of perimodiolar and straight electrode arrays. But we could show that the number of dislocations and of SV insertions depends on the electrode array itself. Furthermore, this study demonstrates that the angular insertion depth negatively impacts on speech perception results. Previous studies showed different results examining the influence of angular insertion depth on postoperative outcome [23-26]. Finley et al. [5] examined 14 patients, implanted with a device from Advanced Bionics $^{\mathrm{TM}}$, and reported that lower outcome scores are associated with greater angular insertion depth and greater number of contacts located in SV. They speculated that the scalar dislocation compromises neural pathways by damaging the basilar membrane and spiral ganglion. Holden et al. [6] $(n=114)$ described that the CNC final score was higher in patients with more electrodes located in ST compared to SV. Finley et al. and Holden et al. [5, 6] speculated that SV inserted electrode arrays can lead to pitch confusion and 


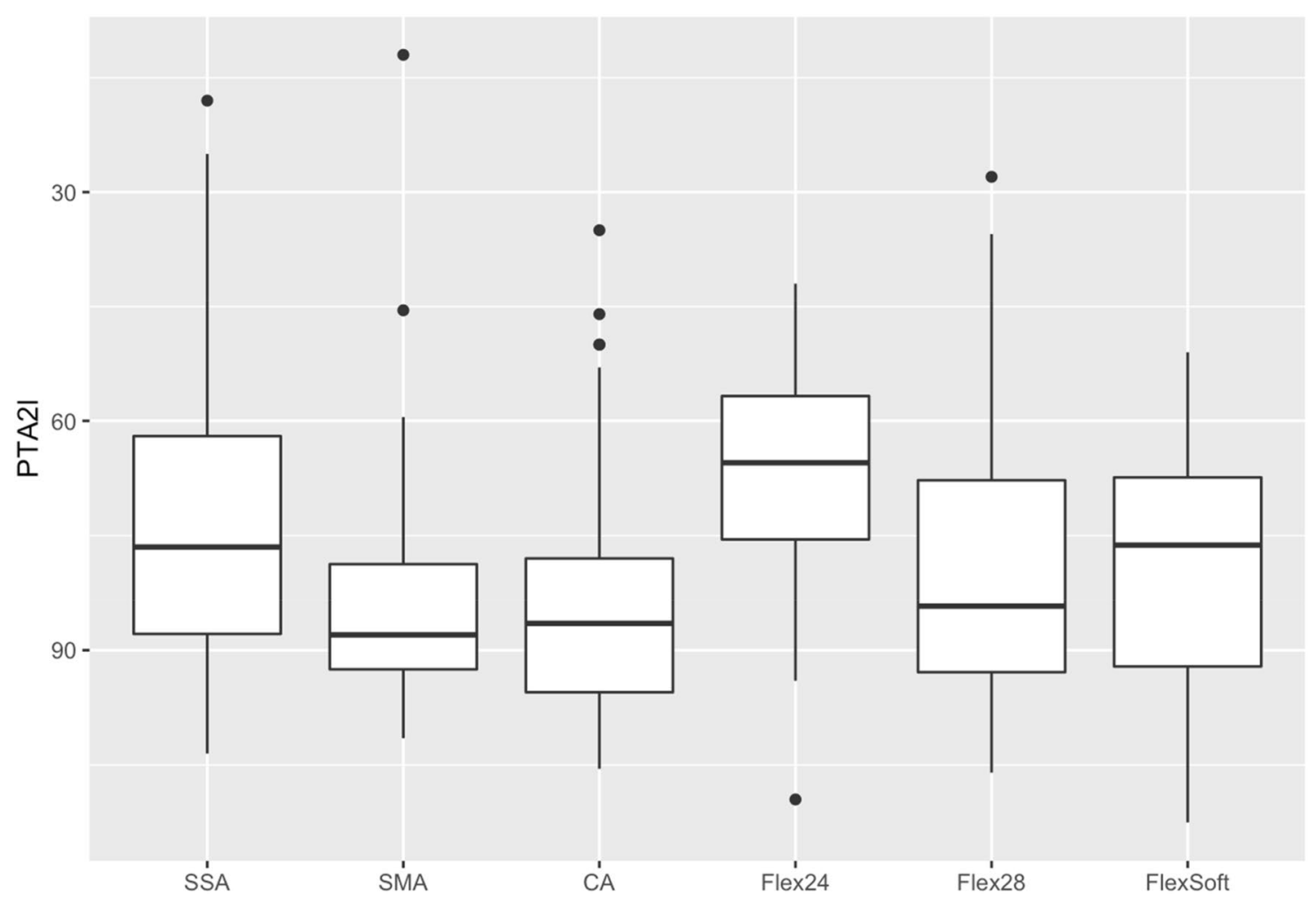

Fig. 6 Preoperative residual hearing levels expressed as PTA $2(250$ and $500 \mathrm{~Hz})$ for each included electrode array

diminished speech recognition due to cross-turn stimulation, but included different types of electrode arrays and did not calculate their results electrode array-specific. Baskent and Shannon [27] examined MED-EL recipients and manipulated electrical stimulation via deactivation of apical electrodes. They described no further benefit for active electrodes over an angular insertion depth of $360^{\circ}$. James et al. [28] collected radiological data via computed tomography and described a negative correlation of angular insertion depth and speech recognition with statistical significance $(p<0.001)$ but detected a very weak correlation $\left(r^{2}=0.09\right)$. However, they included only 96 patients with 9 different electrode arrays. We can now confirm their results regarding the negative impact of increasing angular insertion depth on speech perception in one of the largest cohort studies examining the influence of electrode array design on scalar location, dislocation and the position of dislocation. James et al. [28] described that less insertion depth is associated with better residual hearing preservation [29, 30]. We speculate that the negative correlation of speech perception with increasing angular insertion depth is due to cross-talk of the electric fields in the apical scalae. Nevertheless, there are also other factors to mention, such as the cochlear morphology. As the suspicious lack of publications shows, there is still a lot for speculation and discussion.
The included number of SMA and Flex ${ }^{\text {Soft }}$ arrays in this study was lower compared to the other included electrode arrays and therefore statistical analysis was more difficult. The dislocation rates and SV insertion rates via CS of the included CA group are lower in this study compared to previous studies [28]. Therefore, there might be a sampling bias regarding the influence analysis of scalar dislocation and SV insertion on speech perception results. Further research should be multi-centric to examine more of these electrode arrays. Nevertheless, in conclusion, this is the only study with statistical power and analysis of each electrode array separately, without the bias of electrode arrays differing with respect to length, diameter and rigidity.

Acknowledgements The authors thank the Fördergesellschaft "Taube Kinder lernen Hören e.V.”, which has supported the cochlear implant rehabilitation center in Freiburg for the past several years.

Author contributions MCK, M.D. and RB, M.D. analyzed data, provided statistical analysis and wrote the paper; the other co-authors provided critical revision.

Funding Open Access funding enabled and organized by Projekt DEAL. 


\section{Declarations}

Conflict of interest Manuel Christoph Ketterer and Rainer Beck declare no conflict of interest. Antje Aschendorff received travelling expenses and financial support for research from Cochlear Ltd, Australia; financial support for research and travelling expenses from Med-El, Innsbruck, Austria; financial support for research and travelling expenses from Oticon Inc., Somerset, NJ; financial support for research and travelling expenses from Advanced Bionics, Valencia, CA, USA. Susan Arndt received financial support for research and travelling expenses from Cochlear Ltd, Australia; financial support for research and travelling expenses from Med-El, Innsbruck, Austria travelling expenses from Advanced Bionics, Valencia, CA, USA. This study is not sponsored by industry.

\section{Compliance with ethical standards Yes.}

\section{Research involving human participants No.}

Ethics approval Approved by the Hospital's Ethics Committee according to the Declaration of Helsinki (Washington, 2002) (Number of Ethics Committee approval: 406/19).

Open Access This article is licensed under a Creative Commons Attribution 4.0 International License, which permits use, sharing, adaptation, distribution and reproduction in any medium or format, as long as you give appropriate credit to the original author(s) and the source, provide a link to the Creative Commons licence, and indicate if changes were made. The images or other third party material in this article are included in the article's Creative Commons licence, unless indicated otherwise in a credit line to the material. If material is not included in the article's Creative Commons licence and your intended use is not permitted by statutory regulation or exceeds the permitted use, you will need to obtain permission directly from the copyright holder. To view a copy of this licence, visit http://creativecommons.org/licenses/by/4.0/.

\section{References}

1. Escudé B, James C, Deguine O, Cochard N, Eter E, Fraysse B (2006) The size of the cochlea and predictions of insertion depth angles for cochlea implant electrodes. Audiol Neurootol 11(Suppl 1):27-33

2. Ketterer MC, Aschendorff A, Arndt S, Hassepass F, Wesarg T, Laszig R, Beck R (2018) The influence of cochlear morphology on the final electrode array position. Eur Arch Otorhinolaryngol 275(2):385-394

3. Aschendorff A, Kromeier J, Klenzner T, Laszig R (2007) Quality control after insertion of the nucleus contour and contour advance electrode in adults. Ear Hear 28:75S-79S

4. Skinner MW, Holden TA, Whiting BR, Voie AH, Brunsden B, Neely JG, Saxon EA, Hullar TE, Finley CC (2007) In vivo estimates of the position of advanced bionics electrode arrays in the human cochlea. Ann Otol Rhinol Laryngol Suppl 197:2-24

5. Finley CC, Holden TA, Holden LK, Whiting BR, Chole RA, Neely GJ et al (2008) Role of electrode placement as a contributor to variability in cochlear implant outcomes. Otol Neurotol 29:920-928

6. Holden LK, Finley CC, Firszt JB, Holden TA, Brenner C, Potts LG, Gotter BD, Vanderhoof SS, Mispagel K, Heydebrand G, Skinner MW (2013) Factors affecting open-set word recognition in adults with cochlear implants. Ear Hear 34:342-360

7. Aschendorff A, Kubalek R, Turowski B, Zanella F, Hochmuth A, Schumacher M, Klenzner T, Laszig R (2005) Quality control after cochlear implant surgery by means of rotational tomography. Otol Neurotol 26(1):34-37

8. Ketterer MC, Aschendorff A, Arndt S, Speck I, Rauch AK, Beck R, Hassepass F (2020) Radiological evaluation of a new straight electrode array compared to its precursors. Eur Arch Otorhinolaryngol 278(10):3707-3714

9. Speck I, Ketterer MC, Arndt S, Aschendorff A, Jakob TF, Hassepass F (2021) Comparison of speech recognition and localization ability in single-sided deaf patients implanted with different cochlear implant electrode array designs. Otol Neurotol 42(1): e22-e32

10. Van der Marel K, Briaire JJ, Wolterbeek R, Snel-Bongers J, Verbist BM, Frijns JHM (2013) Diversity in cochlear morphology and its influence on cochlear implant electrode position. Ear Hear 35:9-20

11. Aschendorff A, Klenzner T, Arndt S, Beck R, Schild C, Röddiger L, Maier W, Laszig R (2011) Insertionsergebnisse von Contour $^{\mathrm{TM}}$ - und Contour-Advance ${ }^{\mathrm{TM}}$-Elektroden: Gibt es individuelle Lernkurven? [Insertion results for Contour ${ }^{\mathrm{TM}}$ and Contour Advance $^{\mathrm{TM}}$ electrodes: are there individual learning curves?]. HNO 59(5):448-452 (German)

12. Aschendorff A, Briggs R, Brademann G, Helbig S, Hornung J, Lenarz T, Marx M, Ramos A, Stöver T, Escudé B, James CJ (2017) Clinical investigation of the nucleus slim modiolar electrode. Audiol Neurootol 22(3):169-179

13. Boyer E, Karkas A, Attye A, Lefournier V, Escude B, Schmerber S (2015) Scalar localization by cone-beam computed tomography of cochlear implant carriers: a comparative study between straight and perimodiolar precurved electrode arrays. Otol Neurotol 36(3):422-429

14. Eshraghi AA, Yang NW, Balkany TJ (2003) Comparative study of cochlear damage with three perimodiolar electrode designs. Laryngoscope 113(3):415-419

15. Stöver T, Issing P, Graurock G, Erfurt P, ElBeltagy Y, Paasche G, Lenarz T (2005) Evaluation of the advance off-stylet insertion technique and the cochlear insertion tool in temporal bones. Otol Neurotol 26(6):1161-1170

16. Biedron S, Prescher A, Ilgner J, Westhofen M (2010) The internal dimensions of the cochlear scalae with special reference to cochlear electrode insertion trauma. Otol Neurotol 31(5):731-737

17. Wanna GB, Noble JH, Carlson ML et al (2014) Impact of electrode design and surgical approach on scalar location and cochlear implant outcomes. Laryngoscope 124(Suppl 6 (06)):S1-S7

18. Rebscher SJ, Hetherington A, Bonham B, Wardrop P, Whinney D, Leake PA (2008) Considerations for design of future cochlear implant electrode arrays: electrode array stiffness, size, and depth of insertion. J Rehabil Res Dev 45(5):731-747

19. Souter M, Eastwood H, Marovic P, Kel G, Wongprasartsuk S, Ryan AF, O'Leary SJ (2012) Systemic immunity influences hearing preservation in cochlear implantation. Otol Neurotol 33(4):532-538

20. Hughes ML, Abbas PJ (2006) The relation between electrophysiologic channel interaction and electrode pitch ranking in cochlear implant recipients. J Acoust Soc Am 119(3):1527-1537

21. Müller A, Hocke T, Mir-Salim P (2015) Intraoperative findings on ECAP-measurement: normal or special case? Int J Audiol 54(4):257-264

22. Telmesani LM, Said NM (2015) Effect of cochlear implant electrode array design on auditory nerve and behavioral response in children. Int J Pediatr Otorhinolaryngol 79(5):660-665

23. Buchman CA, Dillon MT, King ER, Adunka MC, Adunka OF, Pillsbury HC (2014) Influence of cochlear implant insertion depth on performance: a prospective randomized trial. Otol Neurotol 35(10):1773-1779

24. Canfarotta MW, Dillon MT, Buchman CA, Buss E, O'Connell BP, Rooth MA, King ER, Pillsbury HC, Adunka OF, Brown 
KD (2021) Long-term influence of electrode array length on speech recognition in cochlear implant users. Laryngoscope 131(4):892-897

25. Canfarotta MW, Dillon MT, Buss E, Pillsbury HC, Brown KD, O'Connell BP (2020) Frequency-to-place mismatch: characterizing variability and the influence on speech perception outcomes in cochlear implant recipients. Ear Hear 41(5):1349-1361

26. Chakravorti S, Noble JH, Gifford RH, Dawant BM, O'Connell BP, Wang J, Labadie RF (2019) Further evidence of the relationship between cochlear implant electrode positioning and hearing outcomes. Otol Neurotol 40(5):617-624

27. Başkent D, Shannon RV (2005) Interactions between cochlear implant electrode insertion depth and frequency-place mapping. J Acoust Soc Am 117(3 Pt 1):1405-1416

28. James CJ, Karoui C, Laborde ML, Lepage B, Molinier CÉ, Tartayre M, Escudé B, Deguine O, Marx M, Fraysse B (2019) Early sentence recognition in adult cochlear implant users. Ear Hear 40(4):905-917
29. Jurawitz MC, Büchner A, Harpel T, Schüssler M, Majdani O, Lesinski-Schiedat A, Lenarz T (2014) Hearingpreservation outcomes with different cochlear implant electrodes: Nucleus ${ }^{\circledR}$ Hybrid $^{\mathrm{TM}}-\mathrm{L} 24$ and Nucleus Freedom ${ }^{\mathrm{TM}}$ CI422. Audiol Neurotol 19(5):293-309

30. Skarzynski H, Lorens A, Matusiak M, Porowski M, Skarzynski PH, James CJ (2014) Cochlear implantation with thenucleus slim straight electrode in subjects with residual low-frequency hearing. Ear Hear 35(2): e33-e43

Publisher's Note Springer Nature remains neutral with regard to jurisdictional claims in published maps and institutional affiliations. 